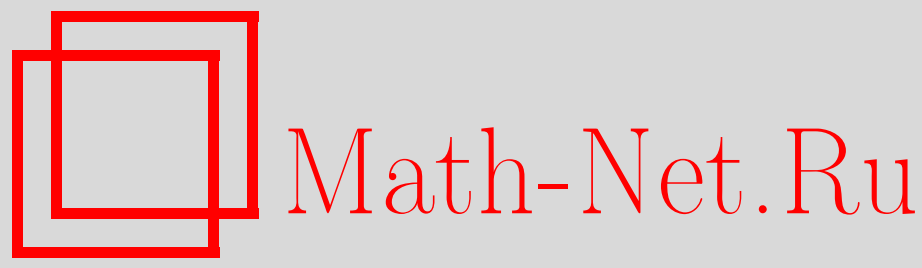

М. В. Павлов, В. Б. Таранов, Г. А. Эль, Обобщенные гидродинамические редукции кинетического уравнения для солитонного газа, ТМФ, 2012, том 171, номер 2, 294302

DOI: https://doi.org/10.4213/tmf6918

Использование Общероссийского математического портала Math-Net.Ru подразумевает, что вы прочитали и согласны с пользовательским соглашением http://www.mathnet.ru/rus/agreement

Параметры загрузки:

IP: 54.209 .52 .79

26 апреля 2023 г., 13:18:18

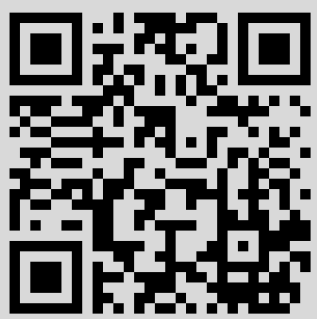


Том 171, № 2

май, 2012

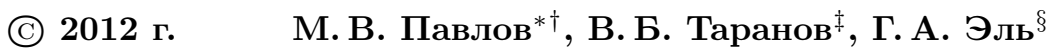

\title{
ОБОБЩЕННЫЕ ГИДРОДИНАМИЧЕСКИЕ РЕДУКЦИИ КИНЕТИЧЕСКОГО УРАВНЕНИЯ ДЛЯ СОЛИТОННОГО ГАЗА
}

\begin{abstract}
Выводятся обобщенные многопотоковые гидродинамические редукции нелокального кинетического уравнения для солитонного газа и исследуется их структура. Эти редукции не только позволяют глубже разобраться в свойствах нового кинетического уравнения, но также могут оказаться представителями нового класса интегрируемых систем гидродинамического типа за пределами общепринятой полугамильтоновой схемы.
\end{abstract}

Ключевые слова: кинетическое уравнение, инварианты Римана, гидродинамическая редукция.

\section{1. ВВЕДЕНИЕ}

Обобщенное кинетическое уравнение для солитонного газа представляет собой интегродифференциальную систему уравнений [1]

$$
\begin{gathered}
f_{t}+(s f)_{x}=0 \\
s(\eta)=S(\eta)+\frac{1}{\eta} \int_{0}^{\infty} G(\eta, \mu) f(\mu)[s(\mu)-s(\eta)] d \mu .
\end{gathered}
$$

Здесь $f(\eta) \equiv f(\eta, x, t)$ - функция распределения и $s(\eta) \equiv s(\eta, x, t)$ - соответствующая скорость переноса. Заданные функции $S(\eta)$ и $G(\eta, \mu)$ не зависят от $x$ и $t$. Функция $G(\eta, \mu)$ предполагается симметричной, т. е. $G(\eta, \mu)=G(\mu, \eta)$.

Система (1), (2), где

$$
S(\eta)=4 \eta^{2}, \quad G(\eta, \mu)=\ln \left|\frac{\eta-\mu}{\eta+\mu}\right|,
$$

${ }^{*}$ Физический институт им. П. Н. Лебедева РАН, Москва, Россия

${ }^{\dagger}$ Московский государственный университет им. М. В. Ломоносова, Москва, Россия. E-mail: maxim@math.sinica.edu.tw

${ }^{\ddagger}$ Институт ядерных исследований НАН Украины, Киев, Украина E-mail: v.taranov@hotmail.ru

${ }^{\S}$ Department of Mathematical Sciences, Loughborough University, Loughborough, United Kingdom. E-mail: g.el@lboro.ac.uk 
выведена в работе [2] как термодинамический предел (когда число зон стремится к бесконечности подходящим образом) уиземовских модуляционных уравнений, связанных с уравнением Кортевега-де Фриза $(\mathrm{Kд} \Phi) \varphi_{t}-6 \varphi \varphi_{x}+\varphi_{x x x}=0$. Показано, что эта система описывает макроскопическую динамику солитонного газа бесконечного набора солитонов, случайно распределенных в пространстве с конечной плотностью [3]. В контексте КдФ $\eta \geqslant 0$ - вещественный спектральный параметр и $f(\eta, x, t)$ - функция распределения солитонов по спектру, таким образом, $\kappa=\int_{0}^{\infty} f(\eta) d \eta=O(1)$ - пространственная плотность солитонов. Если $\kappa \ll 1$, кинетическое уравнение Захарова для разреженного газа солитонов КдФ [4] получается в первом приближении из формул (2), (3). Величина $S(\eta)$ в уравнении (2) имеет естественный смысл скорости изолированного (свободного) солитона со спектральным параметром $\eta$, а функция $G(\eta, \mu) / \eta$ - выражение для фазового сдвига этого солитона, появляющегося после его столкновения с другим солитоном, имеющим спектральный параметр $\mu<\eta$. Тогда $s(\eta, x, t)$ приобретает смысл самосогласованно определенной средней локальной скорости солитонов со спектральным параметром, близким к $\eta$. Прямой физический вывод кинетического уравнения $(1),(2)$ для интегрируемых систем, основанный на использовании фазовых сдвигов солитонов (как это сделано в оригинальной работе Захарова [4]), предложен в работе [5].

В работе [1] многопотоковые гидродинамические редукции кинетического уравнения (1), (2) изучены с помощью так называемого анзаца "холодного газа"

$$
f(\eta, x, t)=\sum_{m=1}^{N} f^{m}(x, t) \delta\left(\eta-\eta^{m}\right)
$$

где "спектральные" компоненты $\eta^{N}>\eta^{N-1}>\cdots>\eta^{1}>0$ - произвольные числа. Было показано, что эти “изоспектральные" редукции холодного газа имеют форму систем гидродинамических законов сохранения:

$$
u_{t}^{i}=\left(u^{i} v^{i}\right)_{x}, \quad i=1, \ldots, N
$$

где сохраняющиеся "плотности" $u^{i}=\eta^{i} f\left(\eta^{i}, x, t\right)$ и соответствующие скорости $v^{i}=$ $-s\left(\eta^{i}, x, t\right)$ связаны алгебраически:

$$
v^{i}=\xi_{i}+\sum_{m \neq i} \epsilon^{i m} u^{m}\left(v^{m}-v^{i}\right), \quad \epsilon^{i k}=\epsilon^{k i} .
$$

Здесь

$$
\xi_{i}=-S\left(\eta^{i}\right), \quad \epsilon^{i k}=\frac{G\left(\eta^{i}, \eta^{k}\right)}{\eta^{i} \eta^{k}}, \quad i \neq k .
$$

В работе [1] доказано, что изоспектральные редукции холодного газа (5), (6) являются интегрируемыми (полугамильтоновыми [6]) слабонелинейными системами гидродинамического типа (см. работы [7], [8]) для произвольного $N$. Это является строгим указанием на то, что полное кинетическое уравнение (1), (2) может оказаться интегрируемым в определенном смысле, в котором еще предстоит разобраться.

Настоящая работа посвящена более общему многопотоковому гидродинамическому приближению кинетического уравнения (1), (2), которое выводится с помощью 
анзаца (см., например, монографию [9])

$$
f(\eta, x, t)=\sum_{m=1}^{N} f^{m}(x, t) \delta\left(\eta-\eta^{m}(x, t)\right)
$$

где "спектральные" компоненты $\eta^{k}=\eta^{k}(x, t)$ являются (неизвестными) функциями от $x$ и $t$ в отличие от произвольных констант в формуле (4). Мы показываем, что соответствующие $N$-потоковые неизоспектральные гидродинамические редукции имеют вид $2 N$-компонентных систем гидродинамического типа

$$
u_{t}^{i}=\left(u^{i} v^{i}\right)_{x}, \quad \eta_{t}^{i}=v^{i} \eta_{x}^{i}, \quad i=1,2, \ldots, N
$$

где функции $u^{i}(x, t), v^{i}(x, t)$ и $\eta^{i}(x, t)$ связаны алгебраически теми же самыми уравнениями $(6),(7)$ при условии, что соблюдены определенные ограничения на поведение функции ядра $G(\eta, \mu)$ при $\eta \rightarrow \mu$.

Система (5), (6), (9) не является интегрируемой стандартным обобщенным методом годографа Царёва, потому что она имеет только $N$ инвариантов Римана и сдвоенные характеристические скорости. Однако, принимая во внимание то, что эта система получена как точная редукция интегрируемой системы (как минимум для $S(\eta), G(\eta, \mu)$, определенных в случае КдФ (3)), можно ожидать, что многопотоковые редукции (9) будут интегрируемыми с помощью некоторой модификации обобщенного метода годографа [6]. Это может привести к расширению общепринятого понятия интегрируемой системы гидродинамического типа. Мы собираемся подробно исследовать эту проблему в дальнейшем.

\section{2. ОБОБЩЕННЫЕ ГИДРОДИНАМИЧЕСКИЕ РЕДУКЦИИ}

2.1. Эволюционные уравнения. Подставляя анзац (8) в уравнение (1), получаем (здесь и далее будем использовать сокращенные обозначения $\eta^{i}$ вместо $\eta^{i}(x, t)$ )

$$
\frac{\partial}{\partial t}\left(\sum_{i=1}^{N} f^{i}(x, t) \delta\left(\eta-\eta^{i}\right)\right)+\frac{\partial}{\partial x}\left(s(\eta, x, t) \sum_{i=1}^{N} f^{i}(x, t) \delta\left(\eta-\eta^{i}\right)\right)=0 .
$$

Дифференцируя и собирая слагаемые с $\delta\left(\eta-\eta^{i}\right)$ и $\delta^{\prime}\left(\eta-\eta^{i}\right)$, получаем

$$
\sum_{n=1}^{N}\left[f_{t}^{n}+\left(s(\eta, x, t) f^{n}\right)_{x}\right] \delta\left(\eta-\eta^{n}\right)-\sum_{n=1}^{N}\left[f^{n} \eta_{t}^{n}+s(\eta, x, t) f^{n} \eta_{x}^{n}\right] \delta^{\prime}\left(\eta-\eta^{n}\right)=0 .
$$

Здесь $f^{i} \equiv f^{i}(x, t)$. Рассматривая асимптотическое поведение этого выражения в окрестности каждой точки $\eta^{i}$, приходим к $2 N$-компонентной системе гидродинамического типа (ср. с формулой (5))

$$
f_{t}^{i}+\left(s\left(\eta^{i}, x, t\right) f^{i}\right)_{x}=0, \quad \eta_{t}^{i}+s\left(\eta^{i}, x, t\right) \eta_{x}^{i}=0, \quad n=1, \ldots, N
$$

Полезно вывести гидродинамическую редукцию (11) прямым вычислением. Она получается интегрированием выражения (10) по $\eta$ в малой окрестности каждой точки $\eta=\eta^{i}$ с весами 1 и $\left(\eta-\eta^{i}\right)$ соответственно. 
Положим $x=x_{0}$. Тогда, выбрав для определенности $\eta^{N}>\eta^{N-1}>\cdots>\eta^{1}>0$, при любых $t$ в малой окрестности $x_{0}$ вводим $N$ замкнутых интервалов $\sigma_{i}=\left[\eta^{i}-\right.$ $\left.\varepsilon_{i}, \eta^{i}+\varepsilon_{i}\right]$, выбирая $\varepsilon_{i}>0$ таким образом, что $\eta^{j}(x, t) \in \sigma_{i}$, если и только если $j=i$ в окрестности $x_{0}$.

Теперь проинтегрируем (10) по интервалу $\sigma_{i}$ :

$$
\int_{\sigma_{i}}\left[\sum_{n=1}^{N}\left[f_{t}^{n}+\left(s(\eta, x, t) f^{n}\right)_{x}\right] \delta\left(\eta-\eta^{n}\right)-\sum_{n=1}^{N}\left[f^{n} \eta_{t}^{n}+s(\eta, x, t) f^{n} \eta_{x}^{n}\right] \delta^{\prime}\left(\eta-\eta^{n}\right)\right] d \eta=0
$$

что после интегрирования слагаемого с множителем $\delta^{\prime}\left(\eta-\eta^{i}\right)$ по частям приводит к

$$
\int_{\sigma_{i}}\left[\sum_{n=1}^{N}\left[f_{t}^{n}+s(\eta, x, t) f_{x}^{n}+\frac{\partial s(\eta, x, t)}{\partial x} f^{n}+\frac{\partial s(\eta, x, t)}{\partial \eta} f^{n} \eta_{x}^{n}\right] \delta\left(\eta-\eta^{n}\right)\right] d \eta=0 .
$$

Далее, интегрирование по интервалу $\sigma_{i}$ немедленно приводит к гидродинамическому закону сохранения

$$
f_{t}^{i}+\left(s\left(\eta^{i}, x, t\right) f^{i}\right)_{x}=0
$$

который верен в малой окрестности $x_{0}$. Если мы будем считать, что вышеприведенные ограничения на поведение функций $\eta^{i}(x, t)$ сохраняются для всех $x=x_{0} \in \mathbb{R}$, $t>0$, то уравнение (13) будет верным на всей вещественной прямой. Полагая $i=1, \ldots, N$ в формуле (12), немедленно получаем первые $N$ уравнений системы (11).

Чтобы вывести вторую половину уравнений (11), умножим выражение (10) на $\left(\eta-\eta^{i}\right)$ и проинтегрируем по интервалу $\sigma_{j}$. Тогда получаем

$$
\begin{aligned}
\int_{\sigma_{j}} & {\left[\sum_{n=1}^{N}\left[f_{t}^{n}+\left(s(\eta, x, t) f^{n}\right)_{x}\right] \delta\left(\eta-\eta^{n}\right)\left(\eta-\eta^{i}\right)\right] d \eta-} \\
& -\int_{\sigma_{j}}\left[\sum_{n=1}^{N}\left[f^{n} \eta_{t}^{n}+s(\eta, x, t) f^{n} \eta_{x}^{n}\right]\left(\eta-\eta^{i}\right) \delta^{\prime}\left(\eta-\eta^{n}\right)\right] d \eta=0 .
\end{aligned}
$$

Если $j=i$, первый из интегралов обращается в нуль, в то время как второй интегрированием по частям приводится к виду

$$
\begin{aligned}
& \int_{\sigma_{i}} \frac{\partial}{\partial \eta}\left(\left(\eta-\eta^{i}\right)\left[f^{i} \eta_{t}^{i}+s(\eta, x, t) f^{i} \eta_{x}^{i}\right]\right) \delta\left(\eta-\eta^{i}\right) d \eta= \\
& \quad=\int_{\sigma_{i}}\left(\left[f^{i} \eta_{t}^{i}+s(\eta, x, t) f^{i} \eta_{x}^{i}\right]+\left(\eta-\eta^{i}\right) \frac{\partial s(\eta, x, t)}{\partial \eta} f^{i} \eta_{x}^{i}\right) \delta\left(\eta-\eta^{i}\right) d \eta=0
\end{aligned}
$$

где мы учли, что каждый интервал $\sigma_{i}$ содержит только “свое” значение $\eta^{i}$. Вычисляя интеграл в формуле (15), получаем

$$
\eta_{t}^{i}+s\left(\eta^{i}, x, t\right) \eta_{x}^{i}=0, \quad i=1, \ldots, N
$$

Нетрудно видеть, что при $j \neq i$ снова получаются уравнения (13). Таким образом, совместимость неизоспектрального анзаца (8) с кинетическим уравнением (1), (2) приводит к уравнениям (16) для функций $\eta^{i}(x, t)$. 
В целом $2 N$-компонентная система гидродинамического типа (11) имеет $N$ законов сохранения

$$
\partial_{t}\left(\varphi_{i}\left(\eta^{i}\right) f^{i}\right)+\left(s\left(\eta^{i}, x, t\right) \varphi_{i}\left(\eta^{i}\right) f^{i}\right)_{x}=0,
$$

где $\varphi_{i}\left(\eta^{i}\right)$ - произвольные функции одной переменной. Для последующих вычислений удобно выбрать $\varphi_{i}\left(\eta^{i}\right)=\eta^{i}$, так что система (11) приводится к виду (ср. с формулой (5))

$$
u_{t}^{i}=\left(u^{i} v^{i}\right)_{x}, \quad \eta_{t}^{i}=v^{i} \eta_{x}^{i}, \quad i=1, \ldots, N
$$

где $u^{i}=\eta^{i} f^{i}, v^{i}=-s\left(\eta^{i}, x, t\right)$.

2.2. Полная система соотношений. Полная система соотношений, связывающая полевые переменные $u^{i}, \eta^{i}$, а также $v^{i}$ в формуле (17), получается подстановкой того же самого анзаца (8) в интегральное уравнение (2). Так как мы собираемся использовать полевые переменные $u^{i}$ вместо $f^{i}$, мы слегка модифицируем анзац (8) следующим образом:

$$
\eta f(\eta, x, t)=\sum_{i=1}^{N} u^{i}(x, t) \delta\left(\eta-\eta^{i}\right) .
$$

Подстановка анзаца (18) в уравнение (2) дает

$$
s(\eta, x, t)=S(\eta)+\sum_{m=1}^{N} u^{m} \frac{G\left(\eta, \eta^{m}\right)}{\eta \eta^{m}}\left[s\left(\eta^{m}, x, t\right)-s(\eta, x, t)\right] .
$$

Так же как и в работе [1], мы вводим (см. формулу (7))

$$
\epsilon^{i k}=\frac{G\left(\eta^{i}, \eta^{k}\right)}{\eta^{i} \eta^{k}}, \quad i \neq k .
$$

Здесь мы сделаем важное замечание. В случае слабонелинейных редукций (5), (6), связанных с изоспектральным анзацем (4), зависимости $\xi^{i}=-S\left(\eta^{i}\right)$ и $\epsilon^{i k}\left(\eta^{i}, \eta^{k}\right)$ от соответствующих компонент вектора $\boldsymbol{\eta}=\left\{\eta^{1}, \eta^{2}, \ldots, \eta^{N}\right\}$ (где $\eta^{i}$ - произвольные константы) не являются важными с точки зрения интегрируемости они только отвечают за связь с исходным кинетическим уравнением (2) (см. работу [1]). Однако в анзаце (8) $\eta^{i}$ - зависимые переменные, $\eta^{i}=\eta^{i}(x, t)$, так что вышеприведенные зависимости становятся существенными для структуры соответствующих гидродинамических редукций.

Теперь мы переходим к пределу при $\eta \rightarrow \eta^{i}$ в выражении (19):

$$
v^{i}=\sum_{m \neq i} \epsilon^{i m} u^{m}\left(v^{i}-v^{m}\right)-S\left(\eta^{i}\right)+\frac{u^{i}}{\left(\eta^{i}\right)^{2}} \lim _{\eta \rightarrow \eta^{i}} G\left(\eta, \eta^{i}\right)\left(s(\eta, x, t)-s\left(\eta^{i}, x, t\right)\right),
$$

где $v^{i}=-\lim _{\eta \rightarrow \eta^{i}} s(\eta, x, t)$ (непрерывность). Если

$$
\lim _{\eta \rightarrow \eta^{i}} G\left(\eta, \eta^{i}\right)\left(s(\eta, x, t)-s\left(\eta^{i}, x, t\right)\right)
$$

существует, то выражение (21) принимает вид

$$
v^{i}=\sum_{m \neq i} \epsilon^{i m} u^{m}\left(v^{i}-v^{m}\right)-S\left(\eta^{i}\right)+g_{i}(\mathbf{u}, \mathbf{v}, \boldsymbol{\eta}),
$$


где

$$
g_{i}(\mathbf{u}, \mathbf{v}, \boldsymbol{\eta})=\frac{u^{i}}{\left(\eta^{i}\right)^{2}} \lim _{\eta \rightarrow \eta^{i}} G\left(\eta, \eta^{i}\right)\left[s(\eta, x, t)-s\left(\eta^{i}, x, t\right)\right] .
$$

Существование предела (22) означает, что функция $G(\eta, \mu)$ имеет особенность не более простого полюса на диагонали $\mu=\eta$.

Если предел (22) зануляется для всех $i=1, \ldots, N$ (что случается, если $G(\eta, \mu)$ либо зануляется, либо имеет особенность слабее чем простой полюс при $\mu \rightarrow \eta$ ), то $g_{i} \equiv 0$ и уравнение (23) приводится к замкнутым условиям (6), (7), полученным для изоспектральной редукции холодного газа. Ниже мы ограничим наше рассмотрение только этим наиболее важным случаем который возникает, в частности, в случае кинетического уравнения для солитонов КдФ [2], когда функция ядра $G(\eta, \mu)$ имеет только логарифмическую особенность на диагонали (см. формулу (3)).

В заключение этого раздела мы отметим, что несуществование предела (22) для некоторой данной функции $G(\mu, \eta)$ означает несовместность дельта-функционального анзаца (18) с интегральным уравнением (2) для соответствующего ядра $G(\mu, \eta)$.

\section{3. СТРУКТУРА ОБОБЩЕННЫХ МНОГОПОТОКОВЫХ ГИДРОДИНАМИЧЕСКИХ РЕДУКЦИЙ}

Используя результаты работы [1] для изоспектральных гидродинамических редукций холодного газа (5), (6), мы вводим симметричную матрицу $\hat{\epsilon}=\left[\epsilon^{m n}\right]_{N \times N}$, где внедиагональные элементы $\epsilon^{i k}(\boldsymbol{\eta})$ определены с помощью соотношений (20), а диагональные элементы $\epsilon^{k k}$ - некоторые новые полевые переменные $r^{k}(\mathbf{u}, \boldsymbol{\eta})$.

Теорема 1 (см. работу [1]). Алгебраическая система (6) допускает параметрическое решение

$$
u^{i}=\sum_{m=1}^{N} \beta_{m i}, \quad v^{i}=\frac{1}{u^{i}} \sum_{m=1}^{N} \xi_{m} \beta_{m i},
$$

где симметрические функции $\beta_{i k}(\mathbf{r}, \boldsymbol{\eta})$ являются элементами матрицы $\hat{\beta}=\left[\beta_{m n}\right]_{N \times N}$ maкой, что $\hat{\beta} \hat{\epsilon}=-\mathbf{1}$.

ДокАЗАТЕЛЬСтво. Заменим (6) эквивалентной системой

$$
v^{i}=\xi_{i}+\sum_{m=1}^{N} \epsilon^{i m} u^{m}\left(v^{m}-v^{i}\right)
$$

(заметим, что суммирование осуществляется по всем $m$, включая $m=i$ (ср. с формулой (6)), которая может быть переписана в виде

$$
v^{i}\left(1+\sum_{m=1}^{N} \epsilon^{i m} u^{m}\right)=\xi_{i}+\sum_{m=1}^{N} \epsilon^{i m} u^{m} v^{m} .
$$

Подставляя выражения (24) в вышеприведенную формулу, получаем

$$
v^{i}\left(1+\sum_{m=1}^{N} \sum_{k=1}^{N} \beta_{m k} \epsilon^{k i}\right)=\xi_{i}+\sum_{m=1}^{N} \sum_{k=1}^{N} \xi_{m} \beta_{m k} \epsilon^{k i} .
$$


Принимая во внимание соотношение $\hat{\beta} \hat{\epsilon}=-\mathbf{1}$, легко видеть, что выражения в обеих частях формулы (26) обращаются в нуль независимо. Таким образом, (26) - тождество, следовательно, параметрическое представление (24) совместно с системой (6). Теорема доказана.

СлеДСТвиЕ 1. Полевые переменные $r^{k}(\mathbf{u}, \boldsymbol{\eta})$ являются рационалъными функииями плотностей законов сохранения $u^{m}$, а именно

$$
r^{k}=-\frac{1}{u^{k}}\left(1+\sum_{m \neq k} u^{m} \epsilon^{m k}(\boldsymbol{\eta})\right), \quad k=1,2, \ldots, N .
$$

В самом деле, умножая обе стороны первого соотношения (24) на $\epsilon^{i k}$ и выполняя суммирование по $i$, получаем

$$
\sum_{m=1}^{N} u^{m} \epsilon^{m k}=\sum_{m=1}^{N} \sum_{n=1}^{N} \beta_{m n} \epsilon^{n k}=-1 .
$$

Таким образом,

$$
\sum_{m \neq k} u^{m} \epsilon^{m k}+r^{k} u^{k}=-1
$$

что немедленно приводит к выражению (27).

ТЕОРема 2. Параметризачия (24) позволяет привести систему гидродинамического типа (17), (6), (7) к блочно-диагональной форме:

$$
\begin{aligned}
\eta_{t}^{i}=v^{i} \eta_{x}^{i}, & i=1, \ldots, N \\
r_{t}^{k}=v^{k} r_{x}^{k}+\frac{1}{u^{k}}\left(\sum_{n \neq k} u^{n}\left(v^{n}-v^{k}\right) \frac{\partial \epsilon^{n k}}{\partial \eta^{k}}+\xi_{k}^{\prime}\right) \eta_{x}^{k}, & k=1, \ldots, N .
\end{aligned}
$$

ДоказАтЕльство. Эволюционные уравнения (28) для функции $\eta^{i}(x, t)$ те же, что и в (17), т. е. необходимо только вывести уравнения (29) для функций $r^{k}(x, t), k=$ $1, \ldots, N$. Подставляя параметрическое представление (24) в законы сохранения (17), получаем

$$
\partial_{t}\left(\sum_{m=1}^{N} \beta_{m i}\right)=\partial_{x}\left(\sum_{m=1}^{N} \xi_{m} \beta_{m i}\right)
$$

Умножая обе стороны на $\epsilon^{i k}$, выполняя суммирование по повторяющемуся индексу $i$ и используя соотношение $\hat{\beta} \hat{\epsilon}=-\mathbf{1}$, получаем уравнения

$$
\sum_{i=1}^{N} \sum_{m=1}^{N} \beta_{m i} \partial_{t} \epsilon^{i k}=\sum_{i=1}^{N} \sum_{m=1}^{N} \xi_{m} \beta_{m i} \partial_{x} \epsilon^{i k}+\partial_{x} \xi_{k}
$$

С учетом очевидного свойства матрицы $\hat{\epsilon}(\mathbf{r}, \boldsymbol{\eta})$

$$
\frac{\partial \epsilon^{n k}}{\partial r^{s}}=\delta_{n k} \delta_{k s}
$$


а также эволюционных уравнений (28) для функций $\eta^{k}$ простое, но не вполне тривиальное вычисление показывает, что уравнения (30) приводятся к виду

$$
r_{t}^{k}=v^{k} r_{x}^{k}+\frac{1}{u^{k}} \sum_{s=1}^{N}\left(\sum_{n=1}^{N} \sum_{m=1}^{N} \beta_{m n}\left(\xi_{m}-v^{s}\right) \frac{\partial \epsilon^{n k}}{\partial \eta^{s}}+\frac{\partial \xi_{k}}{\partial \eta^{s}}\right) \eta_{x}^{s}, \quad k=1, \ldots, N
$$

Так как (см. формулу (24))

$$
\sum_{m=1}^{N} \beta_{m i}=u^{i}, \quad \sum_{m=1}^{N} \xi_{m} \beta_{m i}=u^{i} v^{i}
$$

уравнения (31) приводятся к блочно-диагональному виду (29). Теорема доказана.

Исключая $u^{i}$ из формулы (24), получаем выражения, связывающие $\mathbf{v}$ с $\boldsymbol{\eta}$ и $\mathbf{r}$ :

$$
v^{i}(\mathbf{r}, \boldsymbol{\eta})=\frac{\sum_{m=1}^{N} \xi_{m} \beta_{m i}}{\sum_{m=1}^{N} \beta_{m i}}
$$

в то время как сами $u^{i}(\mathbf{r}, \boldsymbol{\eta})$ определяются из первого уравнения (24). Теперь система (28), (29) стала замкнутой.

В изоспектральном случае, когда $\eta^{i}, i=1,2, \ldots, N,-$ константы (т. е. $\partial_{t} \eta^{i}=$ $\left.\partial_{x} \eta^{i}=0\right)$, из выражения (29) следует представление системы (5), (6) в диагональной форме:

$$
r_{t}^{i}=v^{i}(\mathbf{r}) r_{x}^{i}
$$

найденное в работе [1] благодаря использованию свойства слабонелинейности изоспектральных гидродинамических редукций холодного газа. Фактически теперь можно видеть, что уравнения, записанные в инвариантах Римана (33), могут быть получены прямо из системы (5), (6) с помощью параметризации (24).

Таким образом, $2 N$-компонентная гидродинамическая редукция (17) допускает параметризацию (24) алгебраической системы (6), приводящую эволюционные уравнения к форме (28), (29). Система (28), (29) имеет сдвоенные характеристические скорости $v^{k}(\mathbf{r}, \boldsymbol{\eta})(32)$. Однако в общем случае только $N$ функций $\eta^{k}(x, t)$ являются инвариантами Римана (т.е. только половина полной системы гидродинамического типа (17) может быть записана в диагональной форме), тогда как полевые переменные $r^{k}(x, t)$ становятся инвариантами Римана, только если соответствующие $\eta^{k}=$ const.

В заключение этого раздела заметим, что свойство слабонелинейности системы (33), доказанное в работе [1], означает, что $\partial v^{i}(\mathbf{r}) / \partial r^{i}=0$ для всех $i=1, \ldots N$. Ясно, что последнее свойство остается верным для характеристических скоростей $v^{i}(\mathbf{r}, \boldsymbol{\eta})$ обобщенных гидродинамических редукций $(28),(29)$. Однако теперь оно уже не связано с понятием слабонелинейности систем гидродинамического типа в классическом смысле [10], [11], так как $r^{k}$ не являются больше инвариантами Римана и $\partial v^{i}(\mathbf{r}, \boldsymbol{\eta}) / \partial \eta^{i} \neq 0$.

\section{4. ЗАКЛЮЧЕНИЕ}

В настоящей работе мы вывели обобщенные гидродинамические редукции нелокального кинетического уравнения для солитонного газа $(1),(2)$, используя неизоспектральный многопотоковый анзац (8) для функции распределения. Оказалось, 
что эти новые редукции имеют довольно необычную структуру, которую мы выявили, используя параметризацию (24), примененную к алгебраическим условиям замыкания (6), (7). Точнее, мы показали, что неизоспектральные $N$-потоковые гидродинамические редукции кинетического уравнения представляют собой $2 N$-компонентные полудиагональные системы гидродинамического типа (28), (29) с $N$ инвариантами Римана и $N$ сдвоенными характеристическими скоростями. Хотя выведенные обобщенные гидродинамические редукции, очевидно, не могут быть проинтегрированы обобщенным методом годографа [6], в то же время они могут оказаться интегрируемыми в некотором другом смысле, пока еще не ясном. В самом деле, имея в виду, что система (28), (29) может быть выведена как обобщенная гидродинамическая редукция, связанная с интегрируемым уравнением (т. е. с уравнением КдФ для функций $S(\eta), G(\eta, \mu)$, определенных в $(3))$, можно ожидать, что эта редукция будет интегрируемой некоторым нетривиальным расширением обобщенного метода годографа.

Побочный результат наших вычислений - демонстрация возможности приведения изоспектральных гидродинамических редукций холодного газа к диагональной форме (5)-(7), полученной ранее в работе [1]. Отметим, что приведенный здесь компактный вывод в отличие от использованного в работе [1] не основан на свойствах слабонелинейности изученных гидродинамических редукций.

Благодарности. Авторы благодарят А. Чеснокова за многочисленные дискуссии и полезные консультации. Авторы также признательны М. Жукову, заметившему возможность приведения уравнений (31) к блочно-диагональной форме (29).

Работа М. В. Павлова частично поддержана Правительством РФ (грант № 2010220-01-077), программой президиума РАН “Фундаментальные проблемы нелинейной динамики” и РФФИ (грант № 11-01-00197). М.В.Павлов также благодарен SISSA, Триест (Италия), где была выполнена часть этой работы.

\section{Список литературы}

[1] G. A. El, A. M. Kamchatnov, M. V. Pavlov, S. A. Zykov, J. Nonlinear Sci., 21:2 (2011), 151-191.

[2] G. A. El, Phys. Lett. A, 311:4-5 (2003), 374-383, arXiv: nlin/0310014.

[3] G. A. El, A. L. Krylov, S. A. Molchanov, S. Venakides, Physica D, 152-153 (2001), 653-664, arXiv: nlin/0007025.

[4] В. Е. Захаров, ЖЭТФ, 60:3 (1971), 993-1000.

[5] G. A. El, A. M. Kamchatnov, Phys. Rev. Lett., 95:20 (2005), 204101, 4 pp.

[6] С. П. Царёв, Докл. АН СССР, 282:3 (1985), 534-537; Изв. АН СССР. Сер. матем., 54:5 (1990), 1048-1068.

[7] E. V. Ferapontov, Phys. Lett. A, 158:3-4 (1991), 112-118.

[8] М. В. Павлов, ТМФ, 73:2 (1987), 316-320.

[9] В. П. Силин, Введение в кинетическую теорию газов, Изд-во ФИАН, М., 1971.

[10] P. D. Lax, Commun. Pure Appl. Math., 10:4 (1957), 537-566.

[11] Б. Л. Рождественский, Н. Н. Яненко, Системы квазилинейных уравнений и их приложения в газовой динамике, Наука, М., 1968. 